\title{
ON THE STABILITY OF THE SHEAR-FREE CONDITION
}

\author{
L. Herrera ${ }^{1 *}$ A Di Prisco ${ }^{1 \dagger}$ and J. Ospino ${ }^{2 \ddagger}$ \\ ${ }^{1}$ Escuela de Física, Facultad de Ciencias, \\ Universidad Central de Venezuela, Caracas, Venezuela. \\ ${ }^{2}$ Area de Física Teórica. Facultad de Ciencias,Universidad de Salamanca, \\ Salamanca, Spain.
}

October 2, 2018

\begin{abstract}
The evolution equation for the shear is reobtained for a spherically symmetric anisotropic, viscous dissipative fluid distribution, which allows us to investigate conditions for the stability of the shear-free condition. The specific case of geodesic fluids is considered in detail, showing that the shear-free condition, in this particular case, may be unstable, the departure from the shear-free condition being controlled by the expansion scalar and a single scalar function defined in terms of the anisotropy of the pressure, the shear viscosity and the Weyl tensor or, alternatively, in terms of the anisotropy of the pressure, the dissipative variables and the energy density inhomogeneity.
\end{abstract}

\footnotetext{
*e-mail: laherrera@cantv.net.ve

$\dagger$ e-mail: adiprisc@fisica.ciens.ucv.ve

†e-mail: jhozcrae@usal.es
} 


\section{Introduction}

The relevance of the shear tensor in the evolution of selfgravitating systems and the consequences emerging from its vanishing has been brought out by many authors (see [1]-[6] and references therein). Particular attention deserves the possible role of shear in the violation of the cosmic censorship, leading to the appearance of a naked singularity. This has the important implication that the resultant spacetime singularity of collapse could become visible to faraway observers in the universe (see [4] for a detailed discussion). Furthermore as it has been recently shown [7] the shear-free flow (in the nondissipative case) appears to be equivalent to the well known homologous evolution. It should be recalled that homology conditions are of great relevance in astrophysics [8]-[10].

Accordingly, it is quite pertinent to ask under which conditions the shearfree condition is stable. This question in fact entails two different (but related) questions, namely:

- Under which conditions an initially shear-free flow remains shear-free all along the evolution?

- Under which conditions a fluid with a small initial shear evolves, keeping always a small value of shear (Lyapunov stability)?

Now, in the study of self-gravitating compact objects it is usually assumed that deviations from spherical symmetry are likely to be incidental rather than basic features of the process involved. Thus, since the seminal paper by Oppenheimer and Snyder [11], most of the work dedicated to the problem of general relativistic gravitational collapse, deal with spherically symmetric fluid distribution. Accordingly we shall consider spherically symmetric fluid distributions.

The purpose of this work is to provide answers (at least partial) to the above questions. Obviously for doing so we need an evolution equation for the shear. Such an equation was derived by Ellis [12],[13], for a perfect fluid without any kind of symmetry. Here we shall reobtain that equation for a spherically symmetric anisotropic viscous dissipative fluid. The physical motivation to consider such a general fluid has been explained in detail in [14]-[17] (and references therein). However, since the study of the general 
case is quite complicated and will probably require the use of numerical techniques, we shall consider in detail the case of a geodesic fluid.

Nevertheless, even though the restriction to the geodesic case is dictated by the sake of simplicity in the analysis, the physical relevance of such a case becomes evident when we recall that Friedman-Lemaitre-Robertson-Walker (FLRW) metrics are shear-free. In other words, it appears that for a geodesic fluid (without dissipative fluxes), the stability of the shear-free condition is somehow equivalent to the stability of FLRW spacetime itself.

For the geodesic case it will be shown that departures from the shear-free condition are controlled by the expansion scalar and a scalar function, which is defined in terms of the the Weyl tensor, the anisotropy of pressure and the shear viscosity or, alternatively, in terms of pure physical variables. Such a scalar function appears in a natural way in the orthogonal splitting of the Riemann tensor and is related to the Tolman mass [17]. Together with the expansion scalar, this scalar function controls the evolution of the shear.

In the next section we shall present the general equations and definitions to obtain the evolution equation for the shear. Next in Sec. III that evolution equation is obtained. The origin and some properties of the scalar function which plays such an important role in the evolution of the shear are discussed in Sec.IV, and in Sec. V the obtained equation is used to analyze the geodesic case. Finally, a discussion of results is presented in the last section.

\section{ENERGY-MOMENTUM TENSOR, KINE- MATICAL VARIABLES AND FIELD EQUA- TIONS}

We consider a spherically symmetric distribution of collapsing fluid, bounded by a spherical surface $\Sigma$. The fluid is assumed to be locally anisotropic (principal stresses unequal) and undergoing dissipation in the form of heat flow (to model dissipation in the diffusion approximation), null radiation (to model dissipation in the free streaming approximation) and shearing viscosity.

Choosing comoving coordinates inside $\Sigma$, the general interior metric can be written

$$
d s^{2}=-A^{2} d t^{2}+B^{2} d r^{2}+R^{2}\left(d \theta^{2}+\sin ^{2} \theta d \phi^{2}\right)
$$


where $A, B$ and $R$ are functions of $t$ and $r$ and are assumed positive. We number the coordinates $x^{0}=t, x^{1}=r, x^{2}=\theta$ and $x^{3}=\phi$.

The matter energy-momentum $T_{\alpha \beta}$ inside $\Sigma$ has the form

$$
T_{\alpha \beta}=\left(\mu+P_{\perp}\right) V_{\alpha} V_{\beta}+P_{\perp} g_{\alpha \beta}+\left(P_{r}-P_{\perp}\right) \chi_{\alpha} \chi_{\beta}+q_{\alpha} V_{\beta}+V_{\alpha} q_{\beta}+\epsilon l_{\alpha} l_{\beta}-2 \eta \sigma_{\alpha \beta}
$$

where $\mu$ is the energy density, $P_{r}$ the radial pressure, $P_{\perp}$ the tangential pressure, $q^{\alpha}$ the heat flux, $\epsilon$ the energy density of the null fluid describing dissipation in the free streaming approximation, $\eta$ the shear viscosity coefficient, $V^{\alpha}$ the four velocity of the fluid, $\chi^{\alpha}$ a unit four vector along the radial direction and $l^{\alpha}$ a radial null four vector. These quantities satisfy

$$
V^{\alpha} V_{\alpha}=-1, \quad V^{\alpha} q_{\alpha}=0, \quad \chi^{\alpha} \chi_{\alpha}=1, \quad \chi^{\alpha} V_{\alpha}=0, \quad l^{\alpha} V_{\alpha}=-1, \quad l^{\alpha} l_{\alpha}=0 .
$$

Observe that we have assumed the shear viscosity tensor $\pi_{\alpha \beta}$ to satisfy the relation

$$
\pi_{\alpha \beta}=-2 \eta \sigma_{\alpha \beta},
$$

where $\sigma_{\alpha \beta}$ is the shear tensor. However this last equation is valid only within the context of the standard irreversible thermodynamics (see [18], [19] for details).

In a full causal picture of dissipative variables we should not assume (4). Instead, we should use the transport equation derived from the corresponding theory (e.g. the Müller-Israel-Stewart theory [20]-[22]). However for the sake of simplicity, in this work we shall restrict ourselves to the standard irreversible thermodynamics theory.

The acceleration $a_{\alpha}$ and the expansion $\Theta$ of the fluid are given by

$$
a_{\alpha}=V_{\alpha ; \beta} V^{\beta}, \quad \Theta=V_{; \alpha}^{\alpha} .
$$

and its shear $\sigma_{\alpha \beta}$ by

$$
\sigma_{\alpha \beta}=V_{(\alpha ; \beta)}+a_{(\alpha} V_{\beta)}-\frac{1}{3} \Theta h_{\alpha \beta},
$$

where $h_{\alpha \beta}=g_{\alpha \beta}+V_{\alpha} V_{\beta}$.

We do not explicitly add bulk viscosity to the system because it can be absorbed into the radial and tangential pressures, $P_{r}$ and $P_{\perp}$, of the collapsing fluid [23]. 
Since we assumed the metric (1) comoving then

$$
V^{\alpha}=A^{-1} \delta_{0}^{\alpha}, \quad q^{\alpha}=q B^{-1} \delta_{1}^{\alpha}, \quad l^{\alpha}=A^{-1} \delta_{0}^{\alpha}+B^{-1} \delta_{1}^{\alpha}, \quad \chi^{\alpha}=B^{-1} \delta_{1}^{\alpha},
$$

where $q$ is a function of $t$ and $r$ satisfying $q^{\alpha}=q \chi^{\alpha}$.

From (5) with (7) we have for the acceleration and its scalar $a$,

$$
a_{1}=\frac{A^{\prime}}{A}, \quad a^{2}=a^{\alpha} a_{\alpha}=\left(\frac{A^{\prime}}{A B}\right)^{2},
$$

where $a^{\alpha}=a \chi^{\alpha}$, and for the expansion

$$
\Theta=\frac{1}{A}\left(\frac{\dot{B}}{B}+2 \frac{\dot{R}}{R}\right),
$$

where the prime stands for $r$ differentiation and the dot stands for differentiation with respect to $t$. With (7) we obtain for the shear (6) its non zero components

$$
\sigma_{11}=\frac{2}{3} B^{2} \sigma, \quad \sigma_{22}=\frac{\sigma_{33}}{\sin ^{2} \theta}=-\frac{1}{3} R^{2} \sigma
$$

and its scalar

$$
\sigma^{\alpha \beta} \sigma_{\alpha \beta}=\frac{2}{3} \sigma^{2}
$$

where

$$
\sigma=\frac{1}{A}\left(\frac{\dot{B}}{B}-\frac{\dot{R}}{R}\right) .
$$

Then, the shear tensor can be written as

$$
\sigma_{\alpha \beta}=\sigma\left(\chi_{\alpha} \chi_{\beta}-\frac{1}{3} h_{\alpha \beta}\right)
$$

\subsection{The Einstein equations}

Einstein's field equations for the metric (1) are given by

$$
G_{\alpha \beta}=8 \pi T_{\alpha \beta},
$$


its non zero components with (1), (2) and (7) become

$$
\begin{array}{r}
8 \pi T_{00}=8 \pi(\mu+\epsilon) A^{2}=\left(2 \frac{\dot{B}}{B}+\frac{\dot{R}}{R}\right) \frac{\dot{R}}{R} \\
-\left(\frac{A}{B}\right)^{2}\left[2 \frac{R^{\prime \prime}}{R}+\left(\frac{R^{\prime}}{R}\right)^{2}-2 \frac{B^{\prime}}{B} \frac{R^{\prime}}{R}-\left(\frac{B}{R}\right)^{2}\right], \\
8 \pi T_{01}=-8 \pi(q+\epsilon) A B=-2\left(\frac{\dot{R}^{\prime}}{R}-\frac{\dot{B}}{B} \frac{R^{\prime}}{R}-\frac{\dot{R}}{R} \frac{A^{\prime}}{A}\right), \\
=-\left(\frac{B}{A}\right)^{2}\left[2 \frac{\ddot{R}}{R}-\left(2 \frac{\dot{A}}{A}-\frac{\dot{R}}{R}\right) \frac{\dot{R}}{R}\right] \\
+\left(2 \frac{A^{\prime}}{A}+\frac{R^{\prime}}{R}\right) \frac{R^{\prime}}{R}-\left(\frac{B}{R}\right)^{2}, \\
=-\left(\frac{R}{A}\right)^{2}\left[\frac{\ddot{B}}{B}+\frac{\ddot{R}}{R}-\frac{\dot{A}}{A}\left(\frac{\dot{B}}{B}+\frac{\dot{R}}{R}\right)+\frac{\dot{B}}{B} \frac{\dot{R}}{R}\right] \\
+\left(\frac{R}{B}\right)^{2}\left[\frac{A^{\prime \prime}}{A}+\frac{R^{\prime \prime}}{R}-\frac{A^{\prime}}{A} \frac{B^{\prime}}{B}+\left(\frac{A^{\prime}}{A}-\frac{B^{\prime}}{B}\right) \frac{R^{\prime}}{R}\right] .
\end{array}
$$

\subsection{The mass function}

Let us now introduce the mass function $m(t, r)$ [24] (see also [25]), defined by

$$
m=\frac{R^{3}}{2} R_{23}^{23}=\frac{R}{2}\left[\left(\frac{\dot{R}}{A}\right)^{2}-\left(\frac{R^{\prime}}{B}\right)^{2}+1\right] .
$$

Following Misner and Sharp [24], It is useful to define the proper time derivative $D_{T}$ given by

$$
D_{T}=\frac{1}{A} \frac{\partial}{\partial t}
$$

and the proper radial derivative $D_{R}$,

$$
D_{R}=\frac{1}{R^{\prime}} \frac{\partial}{\partial r},
$$


where $R$ defines the areal radius of a spherical surface inside $\Sigma$ ( as measured from its area).

Using (20) we can define the velocity $U$ of the collapsing fluid as the variation of the areal radius with respect to proper time, i.e.

$$
U=D_{T} R<0 \text { (in the case of collapse). }
$$

Then (19) can be rewritten as

$$
E \equiv \frac{R^{\prime}}{B}=\left(1+U^{2}-\frac{2 m}{R}\right)^{1 / 2} .
$$

Using (15)-(17) with (20) and (21) we obtain from (19)

$$
D_{T} m=-4 \pi\left[\left(\tilde{P}_{r}-\frac{4}{3} \eta \sigma\right) U+\tilde{q} E\right] R^{2},
$$

and

$$
D_{R} m=4 \pi\left(\tilde{\mu}+\tilde{q} \frac{U}{E}\right) R^{2}
$$

which implies

$$
m=4 \pi \int_{0}^{R}\left(\tilde{\mu}+\tilde{q} \frac{U}{E}\right) R^{2} d R,
$$

(assuming a regular centre to the distribution, so $m(0)=0$ ). Integrating (26) we find

$$
\frac{3 m}{R^{3}}=4 \pi \tilde{\mu}-\frac{4 \pi}{R^{3}} \int_{0}^{R} R^{3}\left(D_{R} \tilde{\mu}-3 \tilde{q} \frac{U}{R E}\right) d R .
$$

\section{THE EVOLUTION EQUATION FOR THE SHEAR}

We shall now proceed to deduce the Ellis evolution equation for the shear, for the specific fluid distribution discussed in the previous section. For that purpose it will be convenient to express the energy momentum tensor (2) in the equivalent form

$$
T_{\alpha \beta}=\tilde{\mu} V_{\alpha} V_{\beta}+\hat{P} h_{\alpha \beta}+\Pi_{\alpha \beta}+\tilde{q}\left(V_{\alpha} \chi_{\beta}+\chi_{\alpha} V_{\beta}\right)-2 \eta \sigma_{\alpha \beta}
$$


with

$$
\begin{gathered}
\hat{P}=\frac{\tilde{P}_{r}+2 P_{\perp}}{3}, \\
\tilde{\mu}=\mu+\epsilon, \\
\tilde{P}_{r}=P_{r}+\epsilon, \\
\tilde{q}=q+\epsilon, \\
\Pi=\tilde{P}_{r}-P_{\perp}, \\
\Pi_{\alpha \beta}=\Pi\left(\chi_{\alpha} \chi_{\beta}-\frac{1}{3} h_{\alpha \beta}\right) .
\end{gathered}
$$

Now, Ricci identities for the vector $V_{\alpha}$ read

$$
R_{\alpha \beta \nu}^{\mu} V_{\mu}=V_{\alpha ; \beta ; \nu}-V_{\alpha ; \nu ; \beta},
$$

or, using the well known expression (remember that vorticity vanishes due to the spherical symmetry)

$$
V_{\alpha ; \mu}=-a_{\alpha} V_{\mu}+\sigma_{\alpha \mu}+\frac{1}{3} \Theta h_{\alpha \mu}
$$

we obtain

$$
\frac{1}{2} R_{\alpha \beta \mu}^{\rho} V_{\rho}=a_{\alpha ;[\beta} V_{\mu]}+a_{\alpha} V_{[\mu ; \beta]}+\sigma_{\alpha[\beta ; \mu]}+\frac{1}{3} h_{\alpha[\beta} \Theta_{, \mu]}+\frac{1}{3} \Theta h_{\alpha[\beta ; \mu]} .
$$

Contracting Eq.(31) with $V^{\beta} g^{\alpha \mu}$, we find the Raychaudhuri equation for the evolution of the expansion

$$
\Theta_{; \alpha} V^{\alpha}+\frac{1}{3} \Theta^{2}+\sigma^{\alpha \beta} \sigma_{\alpha \beta}-a_{; \alpha}^{\alpha}=-V_{\rho} V^{\beta} R_{\beta}^{\rho}=-4 \pi(\tilde{\mu}+3 \hat{P}) .
$$

On the other hand, contracting (31) with $V^{\beta} h_{\gamma}^{\alpha} h_{\nu}^{\mu}$ we have

$$
\begin{aligned}
& V^{\beta} V_{\rho} R_{\alpha \beta \mu}^{\rho} h_{\gamma}^{\alpha} h_{\nu}^{\mu}=h_{\gamma}^{\alpha} h_{\nu}^{\mu}\left(a_{\alpha ; \mu}-V^{\beta} \sigma_{\alpha \mu ; \beta}\right)+a_{\gamma} a_{\nu}-\frac{1}{3} V^{\beta} \Theta_{; \beta} h_{\nu \gamma} \\
- & h_{\nu}^{\mu} V_{; \mu}^{\beta}\left(\sigma_{\gamma \beta}+\frac{1}{3} \Theta h_{\gamma \beta}\right)
\end{aligned}
$$


which by using (30), can be written as

$$
\begin{aligned}
V^{\beta} V_{\rho} R_{\alpha \beta \mu}^{\rho} h_{\gamma}^{\alpha} h_{\nu}^{\mu}=h_{\gamma}^{\alpha} h_{\nu}^{\mu}\left(a_{\alpha ; \mu}-V^{\beta} \sigma_{\alpha \mu ; \beta}\right)+a_{\gamma} a_{\nu}-\frac{1}{3} V^{\beta} \Theta_{; \beta} h_{\nu \gamma} \\
-\frac{2}{3} \Theta \sigma_{\gamma \nu}-\frac{\sigma^{2}}{3}\left(\chi_{\gamma} \chi_{\nu}+\frac{1}{3} h_{\gamma \nu}\right)-\frac{1}{9} \Theta^{2} h_{\gamma \nu} .
\end{aligned}
$$

Next, the Riemann tensor may be expressed through the Weyl tensor $C_{\alpha \beta \mu}^{\rho}$, the Ricci tensor $R_{\alpha \beta}$ and the scalar curvature $\mathcal{R}$, as:

$$
\begin{aligned}
R_{\alpha \beta \mu}^{\rho}= & C_{\alpha \beta \mu}^{\rho}+\frac{1}{2} R_{\beta}^{\rho} g_{\alpha \mu}-\frac{1}{2} R_{\alpha \beta} \delta_{\mu}^{\rho}+\frac{1}{2} R_{\alpha \mu} \delta_{\beta}^{\rho} \\
& -\frac{1}{2} R_{\mu}^{\rho} g_{\alpha \beta}-\frac{1}{6} \mathcal{R}\left(\delta_{\beta}^{\rho} g_{\alpha \mu}-g_{\alpha \beta} \delta_{\mu}^{\rho}\right) .
\end{aligned}
$$

Contracting (35) with $V_{\rho} V^{\beta} h_{\gamma}^{\alpha} h_{\nu}^{\mu}$ and using Einstein equations, we find:

$$
V_{\rho} V^{\beta} h_{\gamma}^{\alpha} h_{\nu}^{\mu} R_{\alpha \beta \mu}^{\rho}=E_{\gamma \nu}+\frac{4 \pi}{3}(\tilde{\mu}+3 \hat{P}) h_{\gamma \nu}-4 \pi \Pi_{\gamma \nu}+8 \pi \eta \sigma_{\gamma \nu},
$$

where $E_{\gamma \nu}$ denotes the "electric" part of the Weyl tensor (in the spherically symmetric case the "magnetic" part of the Weyl tensor vanishes), and is defined by

$$
E_{\alpha \beta}=C_{\alpha \mu \beta \nu} V^{\mu} V^{\nu}
$$

whose non trivial components are

$$
\begin{aligned}
& E_{11}=\frac{2}{3} B^{2} \mathcal{E}, \\
& E_{22}=-\frac{1}{3} R^{2} \mathcal{E}, \\
& E_{33}=E_{22} \sin ^{2} \theta,
\end{aligned}
$$

where

$$
\begin{aligned}
\mathcal{E} & =\frac{1}{2 A^{2}}\left[\frac{\ddot{R}}{R}-\frac{\ddot{B}}{B}-\left(\frac{\dot{R}}{R}-\frac{\dot{B}}{B}\right)\left(\frac{\dot{A}}{A}+\frac{\dot{R}}{R}\right)\right] \\
& +\frac{1}{2 B^{2}}\left[\frac{A^{\prime \prime}}{A}-\frac{R^{\prime \prime}}{R}+\left(\frac{B^{\prime}}{B}+\frac{R^{\prime}}{R}\right)\left(\frac{R^{\prime}}{R}-\frac{A^{\prime}}{A}\right)\right]-\frac{1}{2 R^{2}}
\end{aligned}
$$


Observe that the electric part of Weyl tensor, may be written as:

$$
E_{\alpha \beta}=\mathcal{E}\left(\chi_{\alpha} \chi_{\beta}-\frac{1}{3} h_{\alpha \beta}\right) \text {. }
$$

Using (32), (36) can be written as

$$
V_{\rho} V^{\beta} h_{\gamma}^{\alpha} h_{\nu}^{\mu} R_{\alpha \beta \mu}^{\rho}=E_{\gamma \nu}-\left(\frac{1}{3} V^{\alpha} \Theta_{; \alpha}+\frac{\Theta^{2}}{9}+\frac{2}{9} \sigma^{2}-\frac{a_{; \alpha}^{\alpha}}{3}\right) h_{\gamma \nu}-4 \pi \Pi_{\gamma \nu}+8 \pi \eta \sigma_{\gamma \nu},
$$

then, from Eq.(34) and Eq.(41) it follows

$$
\begin{aligned}
& E_{\gamma \nu}-4 \pi \Pi_{\gamma \nu}+8 \pi \eta \sigma_{\gamma \nu}= \\
& h_{\gamma}^{\alpha} h_{\nu}^{\mu}\left(a_{\alpha ; \mu}-V^{\beta} \sigma_{\alpha \mu ; \beta}\right)-\frac{a_{; \alpha}^{\alpha}}{3} h_{\gamma \nu}+a_{\gamma} a_{\nu}-\frac{1}{3} \sigma_{\gamma \nu}(2 \Theta+\sigma)
\end{aligned}
$$

or, using (13)

$$
\begin{aligned}
& E_{\gamma \nu}-4 \pi \Pi_{\gamma \nu}+8 \pi \eta \sigma_{\gamma \nu}= \\
& h_{\gamma}^{\alpha} h_{\nu}^{\mu}\left(a_{\alpha ; \mu}-V^{\beta} \sigma_{\alpha \mu ; \beta}\right)-\frac{a_{; \alpha}^{\alpha}}{3} h_{\gamma \nu}+a_{\gamma} a_{\nu}-\frac{2}{3} \sigma_{\gamma \nu} \Theta+\frac{2}{9} \sigma^{2} h_{\gamma \nu}-\sigma_{\nu}^{\beta} \sigma_{\gamma \beta} .
\end{aligned}
$$

Finally, contracting (43) with $\chi^{\gamma} \chi^{\nu}$ we obtain

$$
\mathcal{E}-4 \pi \Pi+8 \pi \eta \sigma=a^{\dagger}-\sigma^{*}+a^{2}-\frac{\sigma^{2}}{3}-\frac{2}{3} \Theta \sigma-a \frac{R^{\prime}}{R B},
$$

with $f^{\dagger}=f_{, \alpha} \chi^{\alpha}$ and $f^{*}=f_{, \alpha} V^{\alpha}$.

Using (7), (44) takes the form

$$
Y_{T F} \equiv \mathcal{E}-4 \pi \Pi+8 \pi \eta \sigma=\frac{a^{\prime}}{B}-\frac{\dot{\sigma}}{A}+a^{2}-\frac{\sigma^{2}}{3}-\frac{2}{3} \Theta \sigma-a \frac{R^{\prime}}{R B} .
$$

This is the evolution equation for the shear we were looking for (we recall that there is only one independent component of the shear tensor in our case). It is equivalent (in comoving coordinates) to Eq.(71) in [15] or Eq.(101) in [17] (notice that $E$ in those references equals $-\mathcal{E}$ here).

We shall use the above equation to study the stability of the shear-free condition for the case of a geodesic fluid. However before doing that we shall discuss about the origin and the physical properties of $Y_{T F}$. 


\section{ON THE ORIGIN AND PROPERTIES OF $Y_{T F}$}

As we mention in the Introduction, the scalar function $Y_{T F}$ appears in a natural way in the orthogonal splitting of the Riemann tensor (see [17] for details).

Indeed, the electric part of the Riemann tensor (which is one of the element of that splitting) is defined by [26], [27]

$$
Y_{\alpha \beta}=R_{\alpha \gamma \beta \delta} V^{\gamma} V^{\delta}
$$

which in turn can be splitted in terms of its trace and the corresponding trace-free tensor, i.e.

$$
Y_{\alpha \beta}=\frac{1}{3} \operatorname{Tr} Y h_{\alpha \beta}+Y_{<\alpha \beta>}
$$

with $\operatorname{Tr} Y=Y_{\alpha}^{\alpha}$ and,

$$
Y_{<\alpha \beta>}=h_{\alpha}^{\mu} h_{\beta}^{\nu}\left(Y_{\mu \nu}-\frac{\operatorname{Tr} Y}{3} h_{\mu \nu}\right)
$$

this last tensor may also be written as

$$
Y_{<\alpha \beta>}=Y_{T F}\left(\chi_{\alpha} \chi_{\beta}-\frac{1}{3} h_{\alpha \beta}\right) .
$$

To obtain $\operatorname{Tr} Y$ and $Y_{T F}$ we may proceed as in [17] or, directly from (46)(49), using the expressions for the Riemann tensor components in terms of the Einstein tensor components, given in the Appendix of [16]. Either way the result is

$$
\operatorname{Tr} Y \equiv Y_{T}=4 \pi\left(\tilde{\mu}+3 \tilde{P}_{r}-2 \Pi\right)
$$

and

$$
Y_{T F}=4 \pi(-\Pi+2 \eta \sigma)+\mathcal{E} .
$$

Next, using (15), (17), (18) with (19) and (39) we obtain

$$
\frac{3 m}{R^{3}}=4 \pi(\tilde{\mu}-\Pi+2 \eta \sigma)-\mathcal{E},
$$

which combined with (27) and (51) produces 


$$
Y_{T F}=-8 \pi \Pi+16 \pi \eta \sigma+\frac{4 \pi}{R^{3}} \int_{0}^{R} R^{3}\left(D_{R} \tilde{\mu}-3 \tilde{q} \frac{U}{R E}\right) d R .
$$

Thus the scalar $Y_{T F}$ may be expressed through the Weyl tensor and the anisotropy of pressure or in terms of the anisotropy of pressure, the density inhomogeneity and the dissipative variables. It is worth recalling that a link between $Y_{T F}$ and the Tolman mass has been established in [17].

We shall now bring out the role of this scalar function in the stability of the shear-free condition for the geodesic fluid.

\section{THE GEODESIC FLUID}

If we assume the fluid to be geodesic (i.e. $a^{\mu}=0=a$ ) then it follows from (8) and rescaling $t$, that $A=1$. In this case (45) reads

$$
\dot{\sigma}+\frac{\sigma^{2}}{3}+\frac{2 \Theta \sigma}{3}+Y_{T F}=0
$$

where $Y_{T F}$ is defined through (51) or (53).

Let us first assume that $Y_{T F}=0$, then the general solution of (54) is

$$
\sigma(r, t)=\frac{3 c(r)}{\left[c(r) t-e^{\frac{2}{3} \int \Theta d t}\left(1-\frac{2}{3} c(r) \int \Theta t e^{-\frac{2}{3} \int \Theta d \tilde{t}} d t\right)\right]},
$$

where $c(r)$ is a function of integration. After some simple manipulations, this solution can be rewritten as

$$
\sigma(r, t)=\frac{3 c(r) e^{-(2 / 3) \int \Theta d t}}{c(r) \int e^{-(2 / 3) \int \Theta d \tilde{t}} d t-1} .
$$

If we now demand the shear to vanish initially, (i.e. $\sigma(0, r)=0$ ) then as it follows from (55) or (56) we must have (assuming $\Theta$ is a regular function of its arguments)

$$
c(r)=0,
$$

implying $\sigma=0$ for all $t$. Therefore, if $Y_{T F}=0$, the only solution compatible with an initially shear-free flow is a shear-free flow. This result is also evident from (54). 
Before proceeding further, the following remark is in order: Observe that the condition $Y_{T F}=0$ does not imply conformal flatness $(\mathcal{E}=0)$ as it is obvious from (51), unless we assume further the fluid to be perfect. In this latter case the result above is compatible with the one obtained by Stephani [28], [29] stating that the most general conformally flat perfect fluid solution differs from FLRW only by having nonzero acceleration.

Let us now consider the case (always with $Y_{T F}=0$ ) when, initially, the flow is close to the shear-free condition, but still $\sigma \neq 0$. Then the question is under which conditions the fluid will evolve, keeping close to the shear-free regime (Lyapunov stability).

Thus, let us assume

$$
\sigma(0, r)=\epsilon \bar{\sigma}(r), \quad|\epsilon|<<1
$$

Now, for sufficiently small $\epsilon$ and assuming $\Theta$ to be a regular function of $t$, we obtain from (55)

$$
c(r) \approx \mathcal{O}(\epsilon)
$$

implying

$$
\sigma \approx-\epsilon e^{-\frac{2}{3} \int \Theta d t}
$$

From the above it is evident that if $\Theta>0$ then the shear will remain always close to the initial ("quasi-shear-free" condition). However it is also evident that for $\Theta<0$ the situation radically changes and in principle departures from the quasi-shear-free condition may be expected, depending on $\Theta$.

Let us now consider the case $Y_{T F} \neq 0$.

We shall first assume that $Y_{T F}$ (at least initially) is small, i.e.

$$
Y_{T F}=\alpha \bar{Y}_{T F} ; \quad \alpha<<1,
$$

and

$$
\begin{aligned}
\sigma(t, r) & =\sigma_{o}(t, r)+\beta \sigma_{1}(t, r), & \beta<<1 \\
\Theta(t, r) & =\Theta_{o}(t, r)+\xi \Theta_{1}(t, r), \quad & \xi<<1
\end{aligned}
$$

where $\sigma_{o}(t, r)$ and $\Theta_{o}(t, r)$ correspond to the general solution of (54) for $Y_{T F}=0$ given by $(55)$. 
Introducing (62) and (63) into (54) and linearizing we obtain for $\sigma_{1}(t, r)$ the following equation

$$
\dot{\sigma}_{1}+\frac{2}{3}\left(\sigma_{o}+\Theta_{o}\right) \sigma_{1}+\frac{2 \xi}{3 \beta} \Theta_{1} \sigma_{o}+\frac{\alpha}{\beta} \bar{Y}_{T F}=0
$$

which may be easily integrated to obtain

$$
\begin{aligned}
\sigma_{1}(t, r) & =f(r) e^{-(2 / 3) \int\left(\sigma_{o}+\Theta_{o}\right) d t} \\
& -e^{-(2 / 3) \int\left(\sigma_{o}+\Theta_{o}\right) d t} \int \frac{1}{\beta}\left(\frac{2}{3} \xi \Theta_{1} \sigma_{o}+\alpha \bar{Y}_{T F}\right) e^{(2 / 3) \int\left(\sigma_{o}+\Theta_{o}\right) d \tilde{t}} d t,
\end{aligned}
$$

where $f(r)$ is an integration function. Introducing this last equation into (62) we have

$$
\begin{aligned}
\sigma(t, r) & =\sigma_{o}(t, r)+\beta f(r) e^{-(2 / 3) \int\left(\sigma_{o}+\Theta_{o}\right) d t} \\
& -e^{-(2 / 3) \int\left(\sigma_{o}+\Theta_{o}\right) d t} \int\left(\frac{2}{3} \xi \Theta_{1} \sigma_{o}+\alpha \bar{Y}_{T F}\right) e^{(2 / 3) \int\left(\sigma_{o}+\Theta_{o}\right) d \tilde{t}} d t
\end{aligned}
$$

Let us now assume that the fluid is initially shear-free, which implies $\sigma_{0}=0$. Then it follows at once from (66)

$$
\begin{aligned}
\sigma(t, r) & =\beta f(r) e^{-(2 / 3) \int \Theta_{o} d t} \\
& -e^{-(2 / 3) \int \Theta_{o} d t} \int \alpha \bar{Y}_{T F} e^{(2 / 3) \int \Theta_{o} d \tilde{t}} d t
\end{aligned}
$$

If, $Y_{T F}=0(\alpha=0)$ then from the condition of an initially shear-free fluid, we have that $f(r)=0$, implying $\sigma(t, r)=0$ for all $t$, which is the result obtained before.

However, for any $\alpha \neq 0$, it appears from (66) that the fluid may evolve to a non-vanishing shear regime, for any sign of $\Theta$, depending on the specific time dependence of $\bar{Y}_{T F}$. In other words, even for small values of $\alpha$ the fluid may deviate from its initial shear-free condition.

If the fluid initially satisfies the quasi-shear-free condition, which implies that $\sigma_{0}$ is small but non-vanishing. Then in the case $Y_{T F}=\alpha=0$ we obtain 
as before that for $\Theta<0$ departures from the quasi-shear-free condition may be expected, depending on $\Theta$.

In the general $(\alpha \neq 0)$ case however, departures from the quasi-shear-free condition may be expected along the evolution, depending on $Y_{T F}$ for any sign of $\Theta$.

An alternative (and useful) expression for the shear, which allows to study the same problem, may be obtained as follows.

From the geodesic condition

$$
a=0 \Rightarrow A=1
$$

the field equation (16) becomes

$$
\frac{\dot{R}^{\prime}}{R}-\frac{\dot{B}}{B} \frac{R^{\prime}}{R}=4 \pi \tilde{q} B
$$

or,

$$
\frac{\dot{B}}{B}=\frac{\dot{R}^{\prime}}{R^{\prime}}-\frac{F R}{R^{\prime}}
$$

where $F \equiv 4 \pi \tilde{q} B$.

Then, (70) with (9) and (12) produces

$$
2 \Theta+\sigma=3\left(\frac{\dot{R}^{\prime}}{R^{\prime}}+\frac{\dot{R}}{R}-\frac{F R}{R^{\prime}}\right)=3\left(\ln \left(R^{\prime} R\right) \dot{)}-3 \frac{F R}{R^{\prime}}\right.
$$

Introducing (71) into (54) we get

$$
\dot{\sigma}+\left[\left(\ln \left(R^{\prime} R\right) \dot{)}-\frac{F R}{R^{\prime}}\right] \sigma+Y_{T F}=0,\right.
$$

which after integration yields

$$
\sigma=\frac{e^{\int\left(F R / R^{\prime}\right) d t}}{R R^{\prime}}\left(-\int R^{\prime} R Y_{T F} e^{-\int\left(F R / R^{\prime}\right) d \tilde{t}} d t+C(r)\right) .
$$

In the non-dissipative case $(F=0),(73)$ takes the very simple form

$$
\sigma=\frac{1}{R R^{\prime}}\left(-\int R^{\prime} R Y_{T F} d t+C(r)\right) .
$$


Now, if we assume that $Y_{T F}=0$ then it follows at once from (73) that for an initially shear-free fluid we must have $C(r)=0$ implying $\sigma(t, r)=0$.

Also, for an initially quasi-shear-free fluid (with $Y_{T F}=0$ ) it should be clear that departures from that initial condition are possible if $\Theta<0$ and such that it produces a sharp decreasing of $R$ (remember that in order to avoid shell crossing singularities we must have $R^{\prime}>0$ ). Thus we recover our previous result.

In the general case $\left(Y_{T F} \neq 0\right)$ it is obvious from (73) that departures from either the shear-free or the quasi-shear-free initial conditions are possible.

\section{DISCUSSION}

We have carried out a study on the stability of the shear-free condition based on the evolution equation for the shear presented in Sec. III . As shown in the previous section, a major role in such study is played by the scalar $Y_{T F}$. This scalar, which appears in the orthogonal splitting of the Riemann tensor, is not only related with the Tolman mass as shown in [17], but may be expressed through purely physical quantities as in (53).

We shall not insist on the relevance of the question considered in this work (the stability of the shear-free condition) since that was clearly stated in the Introduction. However we would like to comment further on the results concerning the geodesic case.

Indeed, as mentioned before, it is worth noticing that in the non-dissipative, isotropic case, it may be easily shown that (e.g. see [15])

$$
Y_{T F} \equiv \mathcal{E}=0 \Leftrightarrow \sigma=0 \text {. }
$$

Thus for that particular case (geodesic, non-dissipative, isotropic pressure) the shear makes the difference between FLRW metrics $\left(Y_{T F}=\sigma=\mathcal{E}=\right.$ $0)$ and LTB metrics $\left(Y_{T F} \neq 0, \sigma \neq 0\right)$.

Therefore, the discussion in Sec.V illustrates for this particular case, how departures from an initial FLRW spacetime are controlled by $Y_{T F}$.

Finally, it should be emphasized the fact that, even though it may be intuitively obvious that dissipative processes, local anisotropy of pressure and energy density inhomogeneity should affect the stability of the shearfree condition, it is not so obvious that the above mentioned factors affect the stability of the shear-free condition only through their specific combination 
given by (53). Thus for example we could consider the peculiar case when

all those factors are present but they cancel each other in (53), producing $Y_{T F}=0$, in such a case the shear-free condition would be stable in spite of the fact that the fluid is non-homogeneous, non-isotropic and non-dissipative.

\section{Acknowledgments.}

LH and ADP wish to thank Universite Paris VI, France, Universitat de Les Illes Balears, Spain and Universidad de Salamanca, Spain for their hospitality. ADP also wishes to thank Comisión de Investigación and Comisión de Estudios de Posgrado, Facultad de Ciencias, Universidad Central de Venezuela, Venezuela, for finantial support. LH also wishes to thank Fundacion Empresas Polar for finantial support. JO acknowledges financial support from the Universidad de Salamanca (Spain) under grant USAL2008A11.

\section{References}

[1] C. B. Collins and J. Wainwright, Phys. Rev. D 27, 1209 (1983).

[2] E. N. Glass, J. Math. Phys. 20, 1508 (1979).

[3] R. Chan, Mon. Not. R. Astron. Soc. 299, 811 (1998).

[4] P. Joshi, N. Dadhich and R. Maartens, Phys. Rev. D 65, 101501 (2002).

[5] P. Joshi, R. Goswami and N. Dadhich, gr-qc/0308012.

[6] L. Herrera and N. O. Santos, Month. Not. R. Astron. Soc. 343, 1207 (2003).

[7] L. Herrera, N. O. Santos and A. Wang, Phys. Rev. D 78, 084026 (2008).

[8] M. Schwarzschild, Structure and Evolution of the Stars, (Dover, New York) (1958).

[9] R. Kippenhahn and A. Weigert, Stellar Structure and Evolution, (Springer Verlag, Berlin) (1990). 
[10] C. Hansen and S. Kawaler, Stellar Interiors: Physical principles, Structure and Evolution, (Springer Verlag, Berlin) (1994).

[11] J. Oppenheimer and H. Snyder, Phys. Rev. 56, 455 (1939).

[12] G. F. R. Ellis, Relativistic Cosmology in: Proceedings of the International School of Physics "Enrico Fermi", Course 47: General Relativity and Cosmology. Ed. R. K. Sachs (Academic Press, New York and London) (1971).

[13] G. F. R. Ellis, Gen. Rel. Grav. 41, 581 (2009).

[14] L. Herrera and N. O. Santos, Phys. Rep. 286, 53 (1997).

[15] L. Herrera, A. Di Prisco, J. Martín, J. Ospino, N. O. Santos and O. Troconis, Phys. Rev. D 69, 084026 (2004).

[16] A Di Prisco, L. Herrera, G. Le Denmat, M. MacCallum and N.O. Santos, Phys. Rev. D 76, 064017, (2007).

[17] L. Herrera, J. Ospino, A. Di Prisco, E. Fuenmayor and O. Troconis, Phys. Rev. D 79, 064025 (2009)

[18] R. Maartens, astro-ph/9609119.

[19] L. Herrera, A. Di Prisco, E. Fuenmayor and O. Troconis, Int. J. Mod. Phys. D 18, 129, (2009).

[20] I. Müller, Z. Physik 198, 329, (1967).

[21] W. Israel, Ann. Phys., NY 100, 310 (1976).

[22] W. Israel and J. Stewart, Phys. Lett. A58, 213 (1976); Ann. Phys. NY 118, 341 (1979).

[23] R. Chan, L. Herrera and N. O. Santos, Mon. Not. R. Astron. Soc. 267, 637 (1994).

[24] C. Misner and D. Sharp, Phys. Rev. 136, B571 (1964).

[25] M. Cahill and G. McVittie, J. Math. Phys. 11, 1382 (1970). 
[26] L. Bel, Ann. Inst. H Poincaré 17, 37 (1961).

[27] A. García-Parrado Gomez Lobo, arXiv:070\%.1475v2.

[28] H. Stephani, Commun. Math. Phys. 4, 137 (1967).

[29] H Stephani, D Kramer, M MacCallum,C Honselaers and E Herlt, Exact Solutions to Einstein's Field Equations. Second Edition, (Cambridge University Press, Cambridge), (2003) 